\title{
Operation of Robotic Science Boats Using the Telesupervised Adaptive Ocean Sensor Fleet System
}

\author{
Gregg W. Podnar, John M. Dolan, Alberto Elfes, Stephen Stancliff, Ellie Lin, \\ Jeffrey C. Hosler, Troy J. Ames, John Moisan, Tiffany A. Moisan, \\ John Higinbotham, and Eric A. Kulczycki
}

\begin{abstract}
This paper describes a multi-robot science exploration software architecture and system called the Telesupervised Adaptive Ocean Sensor Fleet (TAOSF). TAOSF supervises and coordinates a group of robotic boats, the OASIS platforms, to enable in situ study of phenomena in the ocean/atmosphere interface, as well as on the ocean surface and sub-surface. The OASIS platforms are extended-deployment autonomous ocean surface vessels, whose development is funded separately by the National Oceanic and Atmospheric Administration (NOAA). TAOSF allows a human operator to effectively supervise and coordinate multiple robotic assets using a multi-level autonomy control architecture, where the operating mode of the vehicles ranges from autonomous control to teleoperated human control. TAOSF increases datagathering effectiveness and science return while reducing demands on scientists for robotic asset tasking, control, and monitoring. The first field application chosen for TAOSF is the characterization of Harmful Algal Blooms (HABs). We discuss the overall TAOSF architecture, describe field tests conducted under controlled conditions using rhodamine dye as a HAB simulant, present initial results from these tests, and outline the next steps in the development of TAOSF.
\end{abstract}

\section{INTRODUCTION}

Earth science research requires information obtained from space, the atmosphere and the ocean to foster understanding of the Earth and its natural processes. Developing better models of ocean processes, in particular, is crucial for global

Manuscript received September 14, 2007. This work was supported by NASA award NNX06AF27G, "Telesupervised Adaptive Ocean Sensor Fleet", granted under the Advanced Information Systems Technology program of NASA's Earth Systems Technology Office (ESTO). The TAOSF project is a collaboration among Carnegie Mellon University (CMU), NASA Goddard Space Flight Center (GSFC), NASA Goddard's Wallops Flight Facility (WFF), Emergent Space Technologies, Inc. (EST), and the Jet Propulsion Laboratory (JPL). Work on the OASIS platforms is conducted by Emergent Space Technologies, Inc., EG\&G, and Zinger Enterprises under award NA03NOS4730220 from the National Oceanic and Atmospheric Administration (NOAA), U.S. Department of Commerce.

A. Elfes (corresponding author) is with the Jet Propulsion Laboratory, California Institute of Technology, Pasadena, CA 91109 USA (phone: 818393-6487, fax: 818-393-5007, e-mail: elfes@jpl.nasa.gov).

G. W. Podnar, John M. Dolan, Stephen Stancliff and Ellie Lin are with the Robotics Institute, Carnegie-Mellon University, Pittsburgh, PA 15213 USA (e-mails: gwp@cs.cmu.edu, jmd@cs.cmu.edu, stancliff@cmu.edu, elliel@andrew.cmu.edu).

J. C. Hosler and T. J. Ames are with NASA's Goddard Space Flight Facility, Greenbelt, MD 20771 USA (e-mails: Jeffrey.C.Hosler@nasa.gov, troy.j.ames@nasa.gov).

J. Moisan and T. A. Moisan are with NASA's Wallops Flight Facility, Wallops Island, VA 23337 USA (e-mails: john.moisan@gsfc.nasa.gov, tmoisan@osb1.wff.nasa.gov).

J. Higinbotham is with Emergent Space Technologies, Greenbelt, MD 20770 (e-mail: john.higinbotham@emergentspace.com).

E. A. Kulczycki is with the Jet Propulsion Laboratory, California Institute of Technology, Pasadena, CA 91109 USA (e-mail: eric.a.kulczycki@jpl.nasa.gov). warming, meteorological and ecological studies. Ocean sensing is typically done with satellites, buoys, airborne assets and crewed research vessels. Satellites and airplanes are limited by cloud cover, temporal/geographical coverage, and resolution; while manned research vessels are expensive to deploy, and buoys cannot be self-deployed to specific areas of interest.

The National Oceanic and Atmospheric Administration (NOAA) is addressing some of these constraints through the development of robotic ocean boats for atmosphere-related ocean monitoring. The OASIS (Ocean-Atmosphere Sensor Integration System) vessels are long-duration solar-powered autonomous surface vehicles (ASVs), designed for global open-ocean operations (Figs. 1, 4, 5). One of the key objectives of our research is to enhance the science value of multiple robotic sensing assets, such as the OASIS vessels, by coordinating their operation, adapting their activities in response to sensor observations, and allowing a human operator to oversee multiple assets with minimal effort.

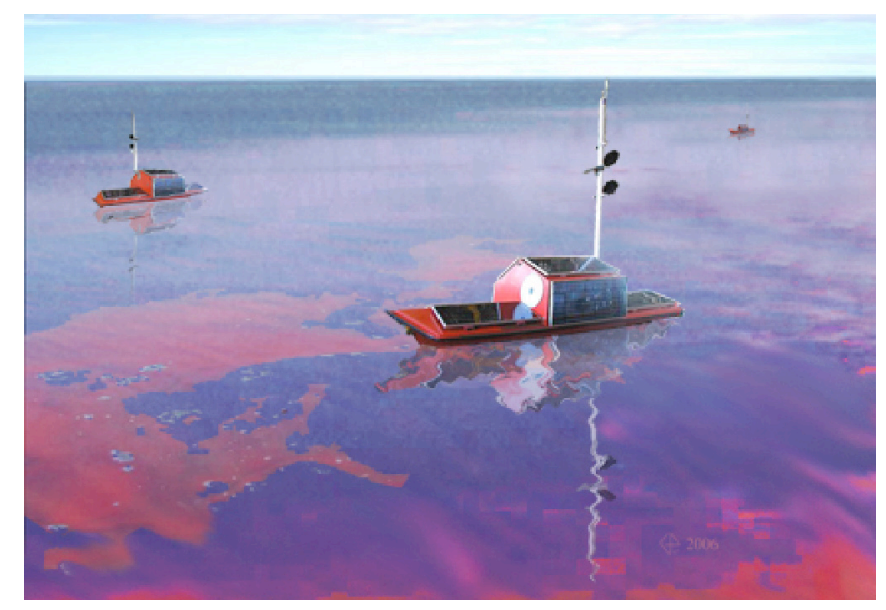

Fig. 1. Artist's concept of the OASIS robot vessels investigating the nature and extent of a Harmful Algal Bloom (HAB).

This paper describes a multi-robot science exploration software architecture and system called the Telesupervised Adaptive Ocean Sensor Fleet (TAOSF). TAOSF is based on the Multilevel Autonomy Robot Telesupervision Architecture (MARTA) [1, 2, 3]. TAOSF supervises and coordinates a group of robotic boats, the OASIS platforms, to enable in situ study of phenomena in the ocean/atmosphere interface, as well as on the ocean surface and sub-surface.

The TAOSF system is applicable to the study of dynamic processes such as coastal pollutants, oil spills, hurricanes or harmful algal blooms. More generally, it could be used in a 
variety of areas where multiple sensing assets are needed, including ecological forecasting, water management, carbon management, disaster management, coastal management, homeland security, and planetary exploration.

The first field application chosen for TAOSF is the characterization of Harmful Algal Blooms (HABs). In the following sections, we will discuss the overall TAOSF architecture, introduce the HAB problem, describe field tests conducted under controlled conditions using rhodamine dye as a HAB simulant, present initial results from these tests, and outline the next steps in the development of TAOSF.

\section{TAOSF ARCHITECTURE}

The TAOSF system architecture (Fig. 2) provides an integrated approach to multi-robot coordination and sliding robot-human autonomy. It allows multiple mobile sensing assets to function in a cooperative fashion, and the operating mode of different vehicles to vary from autonomous control to teleoperated control.

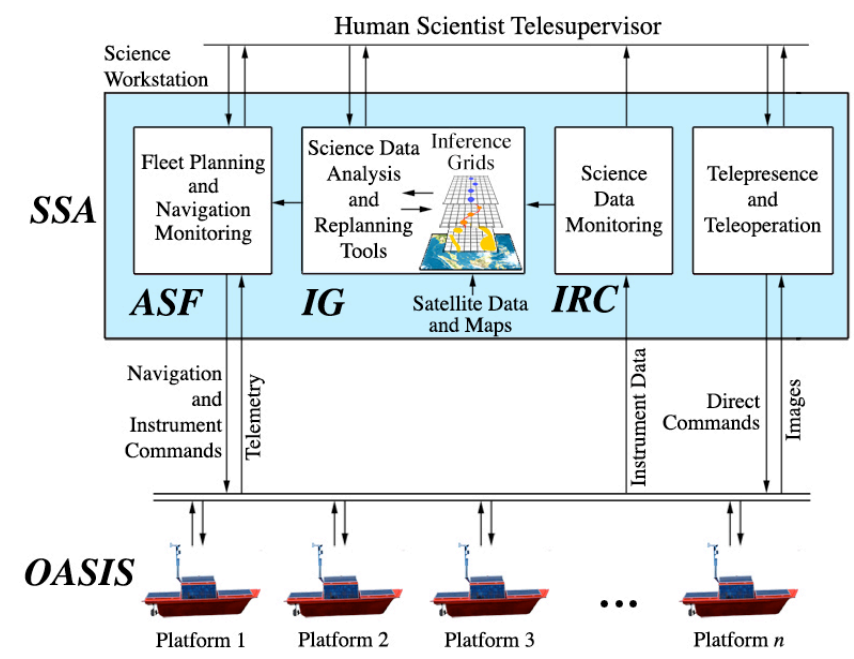

Fig. 2. The TAOSF architecture.

TAOSF supports the following features:

- Multi-level autonomy, allowing an operator to control the vehicles by setting high-level goals, such as specifying an area to monitor, or by taking direct control of the vehicles via teleoperation, or at other autonomy levels in between.

- Adaptive replanning of the activities of the OASIS vessels based on sensor inputs (dynamic sensing) and coordination between multiple assets, thereby increasing data-gathering effectiveness while reducing the effort required for tasking, control, and monitoring of the vehicles.

- Web-based communications permitting control and communications over long distances and the sharing of data with remote experts.

- Autonomous hazard and assistance detection, allowing automatic identification of hazards that require human intervention to ensure the safety and integrity of the robotic vehicles, or of science data that require human interpretation and response.

- Onboard science analysis of the acquired data in order to perform an initial assessment of the presence of specific science signatures of immediate interest.

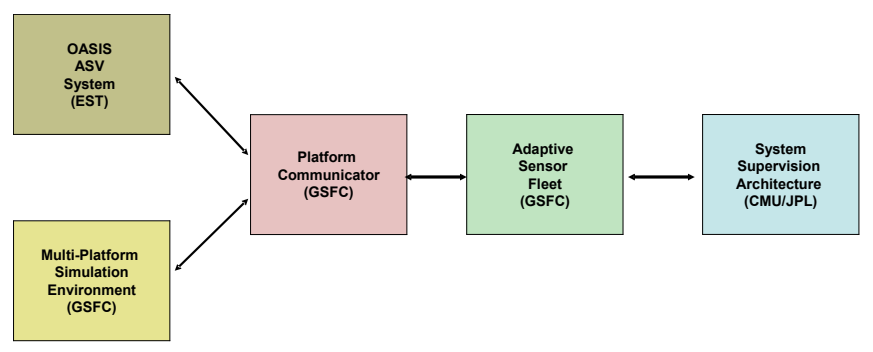

Fig. 3. Software components of the TAOSF architecture.

The TAOSF system software architecture (Fig. 2) integrates and extends five subsystems developed by the participating institutions. The five TAOSF components (Fig. 3) are:

1. The OASIS Autonomous Surface Vehicle (ASV) System includes the vessels themselves, as well as the land-based control and communications infrastructure which has been developed for them. The OASIS platform software directly controls the hardware of each boat (sensors, actuators, etc.), and also provides a lowlevel waypoint navigation capability. This system has been developed by Emergent Space Technologies (EST) working with the team members at the NASA Wallops Flight Facility (WFF), where the platforms are built and maintained.

2. The Multi-Platform Simulation Environment has been developed by NASA Goddard Space Flight Facility (GSFC) as a surrogate for the OASIS ASV system, allowing independent development and testing of the higher-level software components.

3. The Platform Communicator acts as a proxy for both actual and simulated platforms. It translates platformindependent messages from the higher control systems to the device-dependent communication protocols. This component, developed by GSFC, enables the higherlevel control systems to interact identically with heterogeneous actual or simulated platforms.

4. The Adaptive Sensor Fleet (ASF) provides autonomous platform assignment and path planning for area coverage surveys, as well as monitoring of mission progress. The ASF is developed by GSFC.

5. The System Supervision Architecture (SSA) provides high-level planning, monitoring, and telesupervision, as well as analysis of science data from both the OASIS platforms and external sources such as satellite imagery and fixed sensors. These data are used by the SSA to plan vessel navigation trajectories for data gathering. The SSA also provides an operator interface for those occasions when a scientist desires to exert direct monitoring and control of individual boats and their instruments. The SSA is based on the Multilevel Autonomy Robot Telesupervision Architecture (MARTA) developed by CMU and JPL $[1,2,3]$. 
Our initial development effort has concentrated on the integration of these subsystems. We have demonstrated endto-end integration of SSA, ASF and OASIS in a dry-boat test in May of 2007, and executed initial integrated in-water tests in the Chesapeake Bay in June and August of 2007.

\section{OASIS PLATFORMS AND INFRASTRUCTURE}

The NOAA-funded OASIS Platform Build Team, which consists of EG\&G, Zinger Enterprises, and Emergent Space Technologies, provides vehicle development, payload integration and testing, operations, and maintenance of the OASIS fleet and ground systems. The OASIS platform itself is a long-duration solar-powered autonomous surface vehicle (ASV), designed for autonomous global open-ocean operations (Figs. 4, 5). The platform is approximately 18 feet long and weighs just over $3000 \mathrm{lbs}$. The vehicle has a payload capacity of $500 \mathrm{lbs}$, and is designed to be selfrighting for survivability in heavy seas. It supports a wide range of communication links including spread-spectrum radio, a cellular phone link, and an Iridium satellite link.

Three platforms (OASIS1 through OASIS3) are currently undergoing testing at WFF and will support operations for the TAOSF project. Additional platforms are planned. OASIS shakedown operations have been performed since early 2005 in the waters of the DELMARVA region, including the Chincoteague Bay and Pocomoke Sound. The first open-ocean deployment of the OASIS system was performed in November 2006 (Fig. 5). During this operation, the OASIS2 platform successfully navigated over 8 nautical miles on a transect line in the Atlantic Ocean off the coast from WFF. OASIS1 and OASIS2 are currently undergoing upgrades, sensor integration, and testing in preparation for endurance trials and science operations.

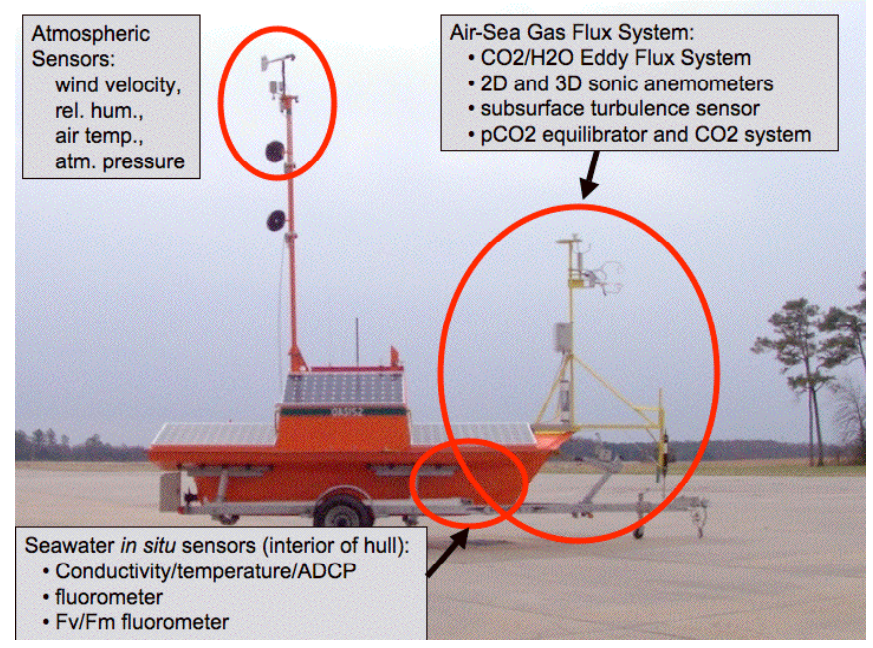

Fig. 4. The OASIS boat with its primary sensor systems.

Sensors onboard the OASIS2 platform enable the collection of water salinity and conductivity data, sea surface temperature, and chlorophyll measurements. A rhodamine fluorometer was integrated to support mapping operations during dye deployment tests. The forward payload bay provides space for installation of additional sensors. This bay includes a water flow-through system with manifolds and a de-bubbling system which simplifies installation of new sensors.

A mast-mounted meteorological station allows acquisition of atmospheric measurements, including barometric pressure, air temperature, relative humidity, wind speed, and wind direction. OASIS2 is also equipped with a forwardlooking digital imaging system providing remotely located scientists with images of atmospheric and sea state conditions.

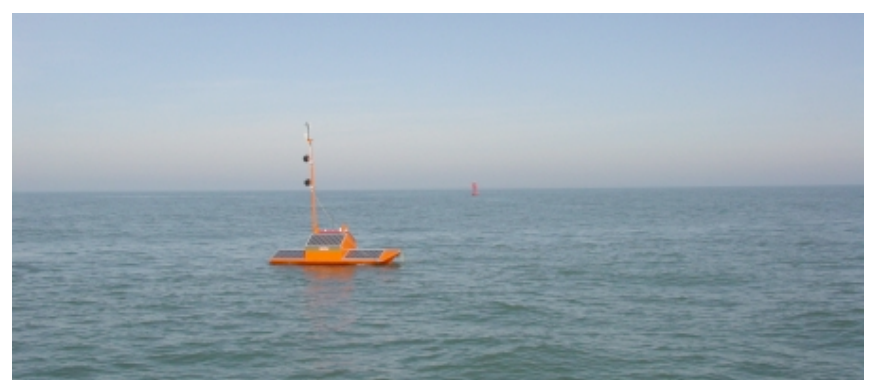

Fig. 5. First OASIS open-ocean deployment, November 2006.

The off-board infrastructure developed by EST is known as the OASIS Mission Operations Environment (MOE). The MOE resides in the Wallops Coastal Ocean Observation Laboratory (WaCOOL) control room and provides applications and services that enable the WFF engineering and science operations team to perform platform commanding and telemetry monitoring, as well as communications management. The MOE also provides a middleware interface to enable other customers, such as the TAOSF project, to integrate new systems that further enhance OASIS science operations.

\section{HARMFUl Algal BloOMS}

Interest in Harmful Algal Bloom (HAB) detection has grown in recent years for scientific, commercial and public health reasons. Depending on the type of algae present, HABs have been shown to be dangerous to sea life and to human health.

There is a significant interest in identifying environmental factors that contribute to the occurrence of HABs, so that these may be incorporated in bloom prediction algorithms [4, $5,6]$. A regional study on the dinoflagellate Karlodinium veneficum has been generating near-real-time maps of HABs in the Chesapeake Bay using a hydrodynamic model and satellite data [5]. The methodology uses time of year, salinity, and sea-surface temperature to predict the abundance (low, medium, or high) of the dinoflagellate.

TAOSF will provide the following advantages over existing systems for observing and analyzing HABs:

- Dynamic tasking and adaptation

- Higher in situ resolution and greater insensitivity to cloud cover as compared with satellite systems

- Access to and greater agility in coastal waters than what is available through buoys

- Real-time multipoint science data observations and generation of associated interpretations by remotely located oceanographers. 


\section{HAB SENSING AND CHARACTERIZATION}

Our work in this area has two components [7, 8]. First, we are assembling and analyzing all known HAB-related data from the Chesapeake Bay area. Second, for initial sensor testing and validation we have developed a means of producing and ground-truthing a surrogate $\mathrm{HAB}$ using rhodamine, a fluorescent compound commonly used as a water tracer dye.

The Maryland Department of Natural Resources (DNR) has provided us with descriptions and HAB species cellcount data from five regions in the Chesapeake and Coastal Bays that have experienced algal blooms. We used a Gaussian process approach to predict the cell counts of the dinoflagellate Karlodinium micrum from water quality features (temperature, salinity, and dissolved oxygen). The results are beyond the scope of this paper, and are discussed in $[7,8]$. We also investigated an adaptive sampling approach using the Regional Ocean Modeling System (ROMS) model of the Chesapeake Bay using Gaussian processes to select positions for obtaining sensor measurements to optimally characterize the distribution of salinity from known temperature data $[7,8]$.

The next step in our analysis will be to integrate MODIS (Moderate Resolution Imaging Spectroradiometer) satellite data (Chlorophyll A and sea surface temperature) with the DNR cell-count data for $\mathrm{HAB}$ prediction.

Since HABs are sporadic ocean phenomena, we are using rhodamine WT (water-tracing) dye [9] as a HAB surrogate for initial experiments and tests. Because some of the TAOSF system testing and validation is done using an aerial imaging platform (see section VI), it became necessary to correlate aerial dye visibility to dye concentration in the water. This was done by imaging dye diffusion and visibility in a local pond in Pittsburgh.

The pond, called Schenley Park Lake, has a surface area of about $9900 \mathrm{~m}^{2}$, and a depth conservatively estimated at 1 $\mathrm{m}$. Dye was introduced at two locations in the lake, and the dye patch dispersions were tracked visually. Surface water samples were taken of each patch at measured time intervals and were analyzed using the same fluorometers used on the OASIS platform. This allowed us to establish a baseline for correlating camera visibility with in-water concentration measurements $[7,8]$.

In the TAOSF system, we use the Inference Grid (IG) model to represent multiple spatially- and temporallyvarying properties. The Inference Grid is a probabilistic multi-property spatial lattice model (a Markov Random Lattice) $[10,11]$, where sensor information is stored in spatially and temporally registered form, and which is used for both scientific inferences and vehicle mission planning. The information in each Inference Grid cell is represented as a stochastic vector, and metrics such as entropy are used to measure the uncertainty in the IG $[12,13]$.

As part of the HAB sensing and characterization effort, we have developed an initial automated dye patch mapping system using the IG model. Overhead imagery of the pond was processed to perform automated cluster identification using an unsupervised clustering algorithm. Using a reference dye spectral signature, the dye dispersion patch was identified and segmented in the image, and a probabilistic metric was associated with the distance to the reference signature in the spectral space. The results for a single pond image are shown in Fig. 6, and for a sequence of images in Fig. 7.

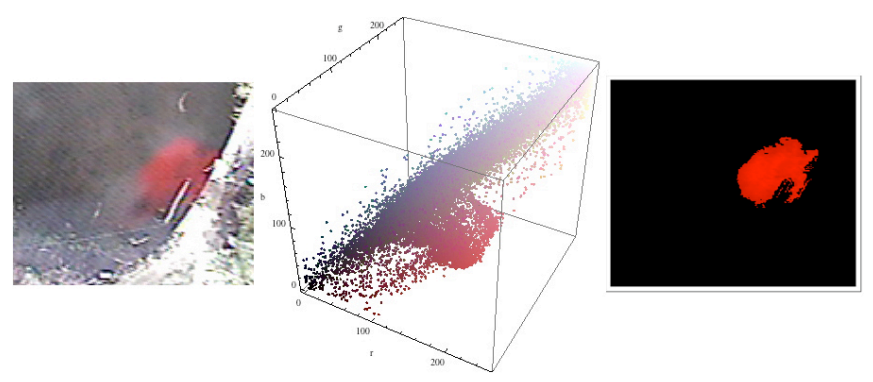

Fig. 6. The left image shows a dye patch after several minutes of dye dispersion. The center plot shows the RGB intensity distributions of the left image; automated image segmentation is done using an unsupervised clustering algorithm. The map on the right shows an Inference Grid with the spatially distributed probabilities of dye being present in the water.

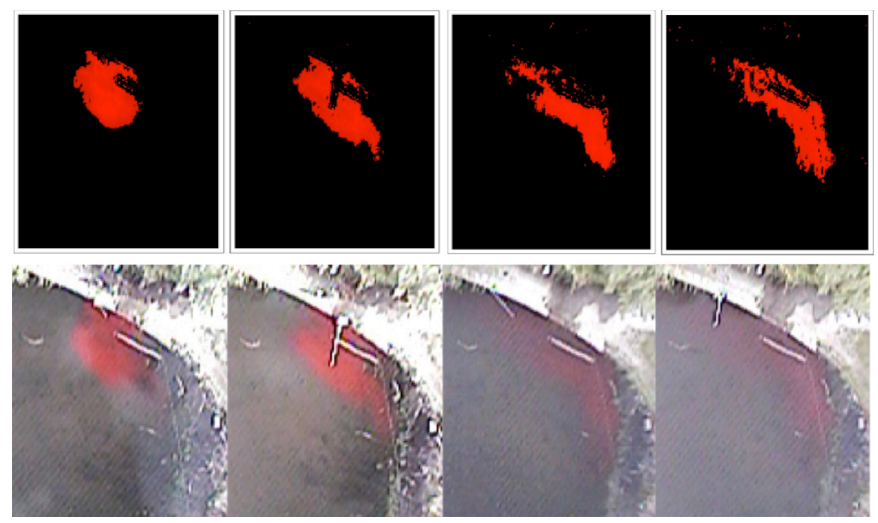

Fig. 7. Automated dye patch extent mapping and characterization using the Inference Grid. The images in the bottom row show a dye patch dispersing over time. The evolution of the corresponding Inference Grid is shown in the upper row, where the spatially and temporally varying dye presence probabilities are shown.

\section{AERIAL Field TeSt ObSERVATION PlatForm}

One important component in the field testing and validation process of the overall TAOSF system is the ability to have a "bird's eye" view of how an algal bloom (or a dye patch) is moving and dispersing in the water, and how the OASIS boats are responding to this process. To address this need, we developed an aerial observation system carrying an avionics package that includes GPS, barometric altimeter, magnetic compass, serial data link, wide-angle color camera, and video transmitter $[7,8]$. Fig. 8 depicts the typical deployment and testing configuration, with the OASIS platforms investigating a dye-simulated bloom, and an aerostat carrying the aerial observation system while tethered to a human-piloted chase boat.

The TAOSF aerial observation platform is a $6 \mathrm{~m}$ long, $2 \mathrm{~m}$ diameter aerostat (Fig. 9). The aerostat is an unmanned, helium-filled, lighter-than-air (LTA) blimp on a tether. In its current configuration the system is flown unpowered. The onboard avionics package (Fig. 10) is gimballed, with a total weight of $700 \mathrm{~g}$. 


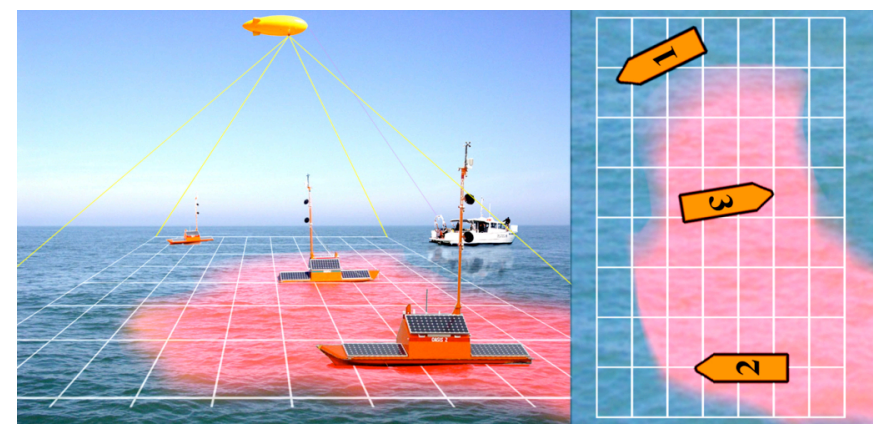

Fig. 8. Concept of the TAOSF field validation system: an overhead aerostat (an unmanned blimp tethered to a manned field operations vessel) provides a global visual overview of three OASIS platforms and a patch of rhodamine dye. The overhead map is shown on the right.

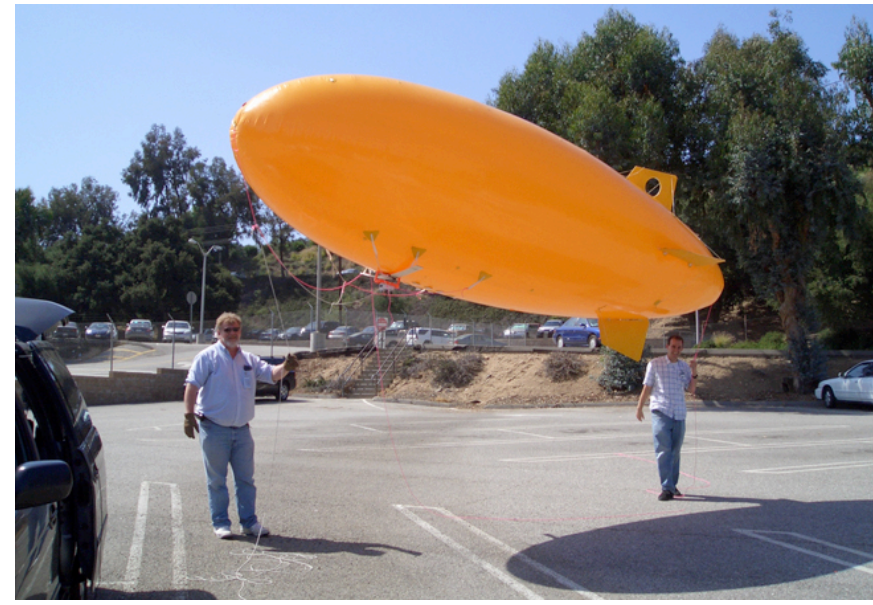

Fig. 9. The TAOSF aerostat observation platform. The aerostat is an unmanned, lighter-than-air (LTA) unpowered blimp on a tether. Helium is used for buoyancy.

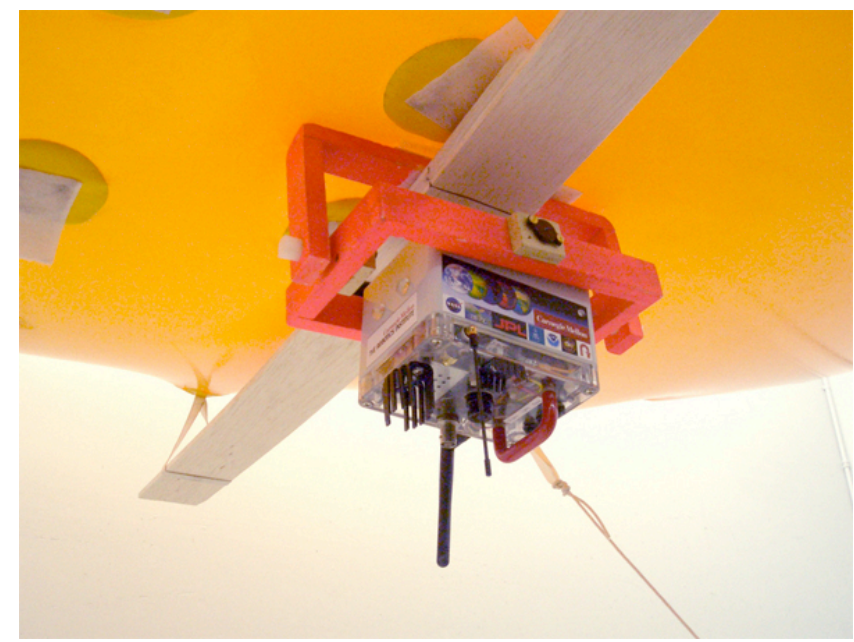

Fig. 10. The gimballed TAOSF observation platform avionics package, below the aerostat, includes a GPS, a barometric altimeter, a magnetic compass, a serial wireless data link, a wideangle color camera, and a video transmitter.

We emphasize that the aerial observation platform is currently not being used to help identify and track HABs; rather, its purpose is to provide a global view of our field tests to assess the in situ performance of the TAOSF system.

\section{INTEGRATED SYSTEM TESTING AND VALIDATION}

The first integrated in-water tests of the TAOSF architecture were conducted on 27 June and 21 August, 2007. Here, we review the 21 August test, where a single OASIS vessel was used, the aerial observation platform was deployed, and rhodamine dye tracks were laid by a manned chase boat (Fig. 11).

A forward view from the OASIS camera was displayed on the remote operator workstation (Fig. 12), allowing teleoperation of the boat if necessary. The rhodamine tracks laid are shown in Fig. 13.
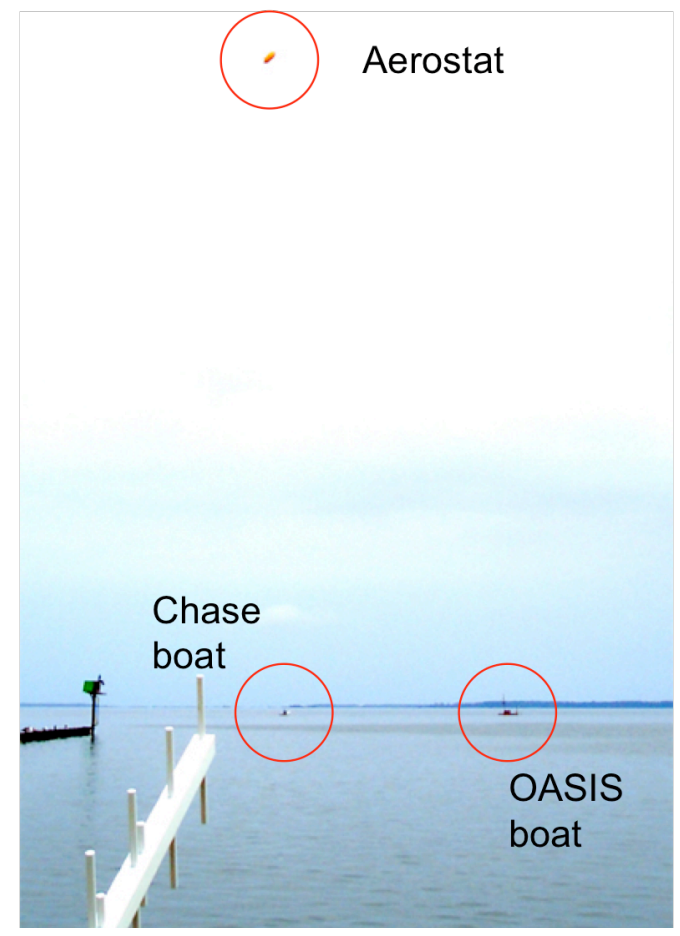

Fig. 11. Scenario of the August 21 test. The TAOSF aerostat can be seen above the chase boat and one OASIS platform. The photo gives a fair sense of scale of the operation, conducted about one mile from shore.

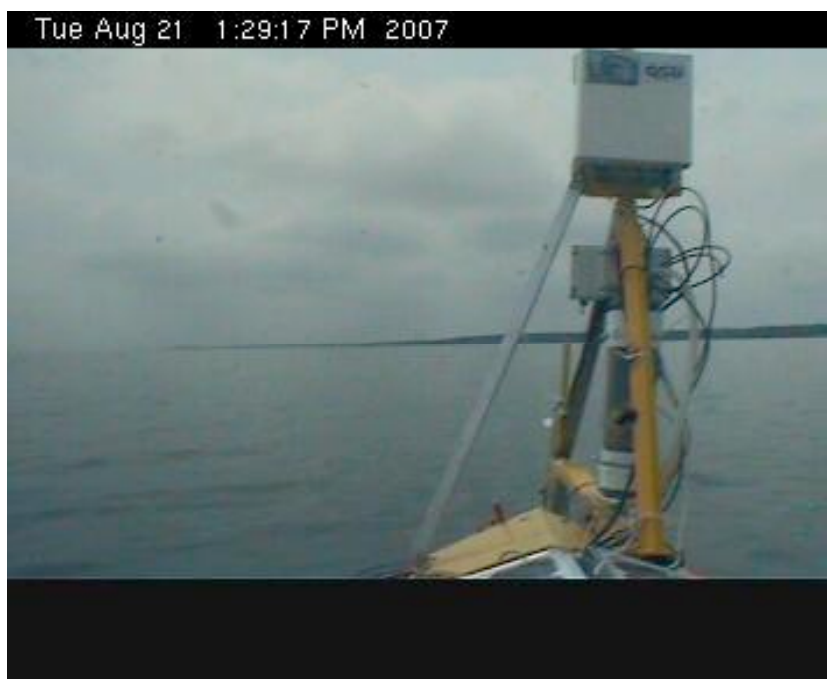

Fig. 12. Forward view from the OASIS boat, as seen from the remote operator workstation. 


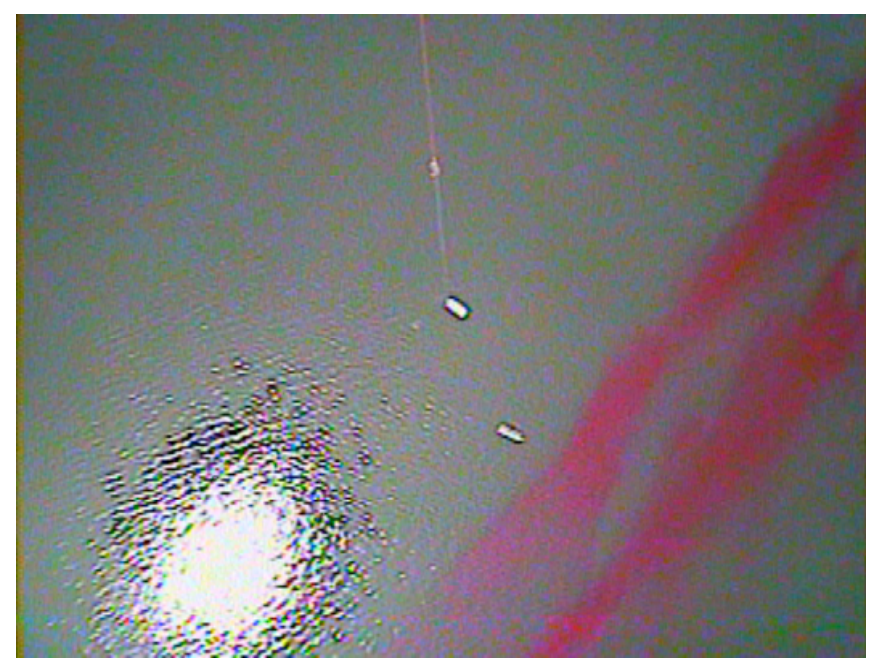

Fig. 13. Aerial view from the aerostat observation platform. The chase boat (with aerostat tether) is in the center of the image, the OASIS platform below it, and the rhodamine dye tracks laid by the chase boat can be seen on the right.

A spiral search pattern was planned and executed by the ASF component of the TAOSF system (Figs. $14-16$ ). The operator interface (Fig. 14), based in part on the Multi-Robot Operator Control Unit (MOCU) [14], displays the boat trajectory, telemetry and position within a satellite map. The trajectories of the OASIS boat and the aerostat observation platform are shown in Fig. 15, and the measured rhodamine dye concentrations along the search path in Fig. 16.

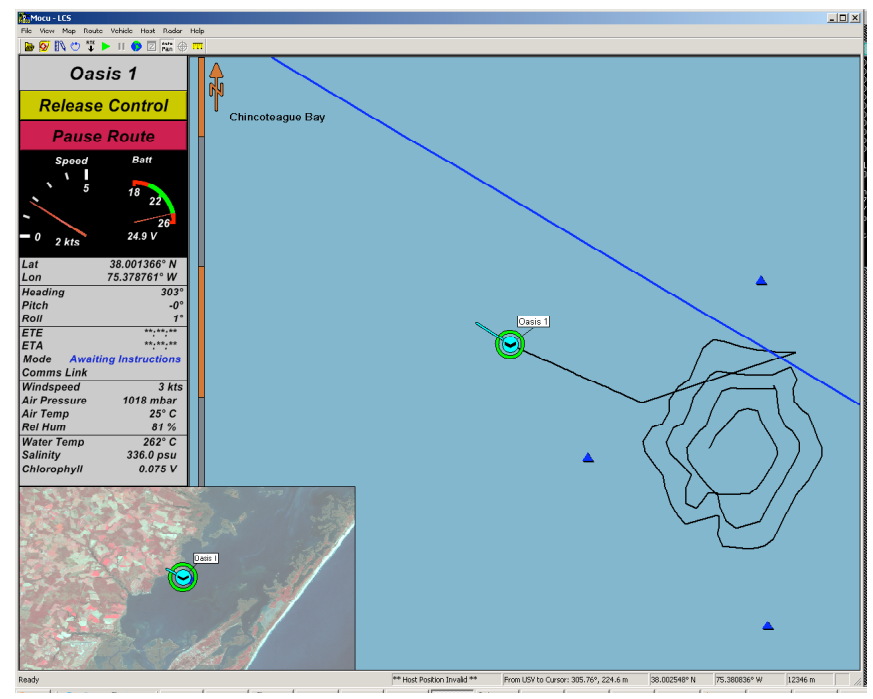

Fig. 14. The Multi-Robot Operator Control Unit (MOCU), part of SSA. The right pane shows the spiral search pattern planned and executed by the ASF component of TAOSF. The upper left pane displays the engineering and science telemetry from the OASIS boat, while the lower left pane shows the position of the OASIS platform overlaid on a satellite view of the operating area.

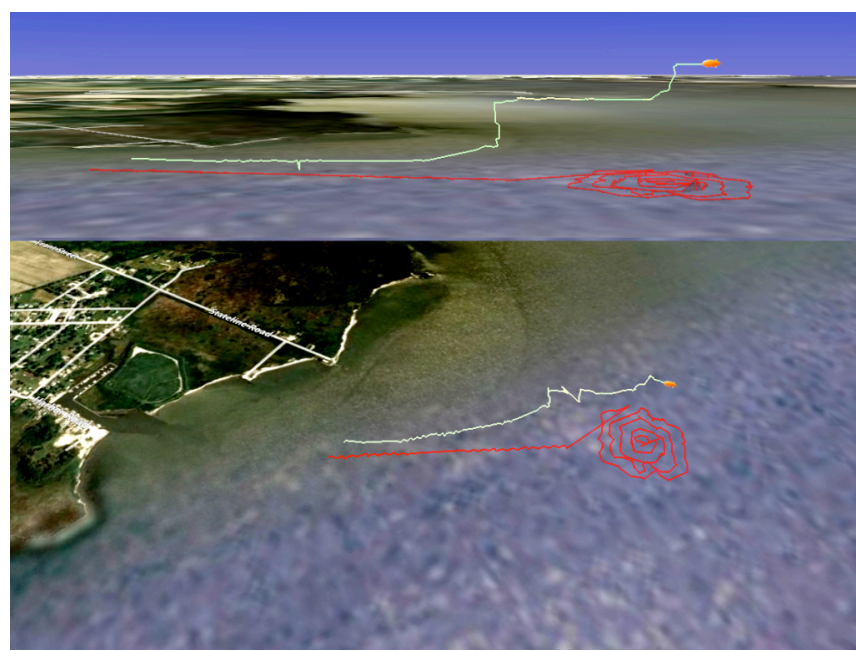

Fig. 15. Two angles of a satellite view of the August 21 field test area. The GPS track of the OASIS boat is shown in red, while the aerostat observation path (obtained from the onboard avionics) is shown in white.

\section{HAB MAPPING RESULTS}

The rhodamine dye concentration measurements taken by the OASIS platform were used as input to the Inference Grid mapping process. One of the layers we have defined in the Inference Grid represents the spatially distributed probabilistic estimates of the presence of a specific type of $\mathrm{HAB}$; in the case of our August 21 test, the layer is actually used to estimate the presence of rhodamine dye. For this particular test, the goal was just to determine the presence or absence of dye in the water, and to infer possible dye tracks from the data. For simplicity, we assumed that both the dispersion of dye in the water and the ocean currents would be slow enough to preclude the need to estimate the dynamics of the dye dispersion and transport processes. This assumption will be relaxed in future tests.

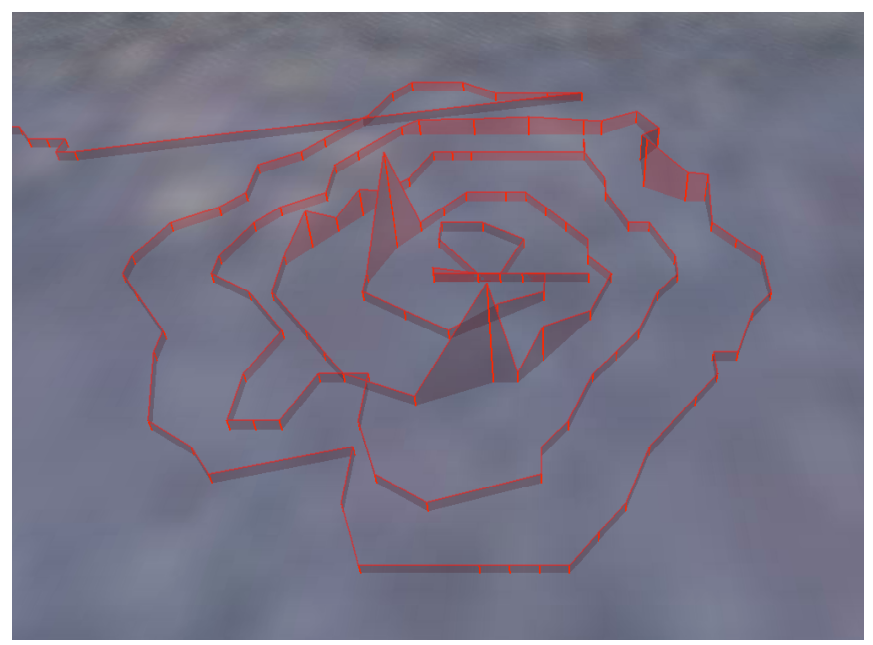

Fig. 16. A slanted view of the ocean area where the test was conducted. The measured rhodamine concentrations are shown as a vertical "ribbon" along the route taken by the OASIS boat (as shown in Figs. 14 and 15). 
For Inference Grid estimation, the region of interest investigated by the OASIS boat was divided into cells with a spatial size of $10^{-4}$ longitudinal degrees $\times 10^{-4}$ latitudinal degrees (roughly $10 \mathrm{~m} \times 11 \mathrm{~m}$ at the Chesapeake Bay). The dye measurements (Fig. 16) were used to compute the probabilistic estimates of the presence or absence of dye for each cell in the area traversed by the OASIS boat. The probabilistic sensor model was derived from information on the sensitivity and performance of the fluorometers onboard OASIS. The Inference Grid map of rhodamine dye presence (the probabilistic dye map, or PDM) is shown in Fig. 17.

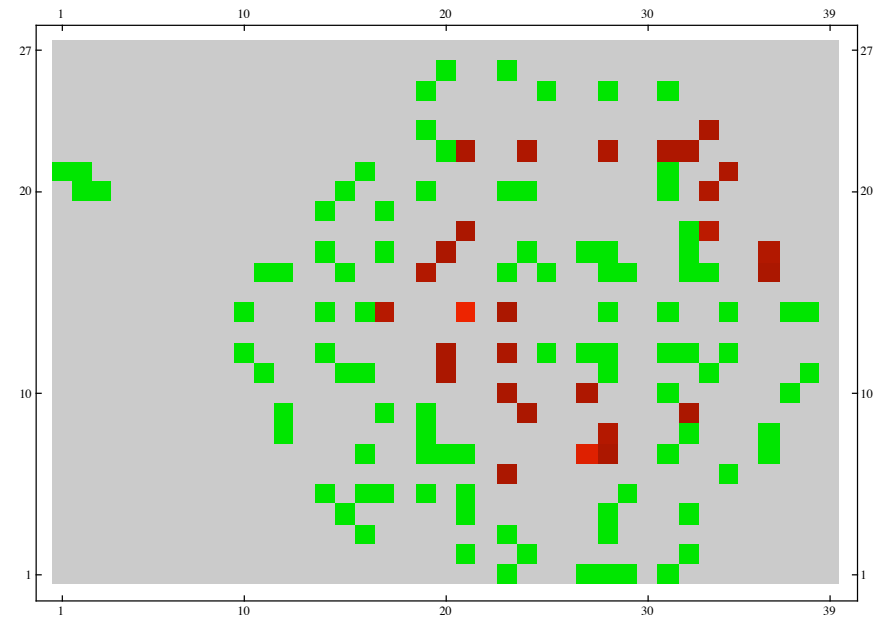

Fig. 17. The Probabilistic Dye Map (PDM). This is the Inference Grid map layer showing probabilistic estimates of the presence of rhodamine dye in the region traversed by the OASIS boat. Green cells indicate high probability of dye absence, red cells indicate high probability of dye presence, and grey cells indicate regions where no measurements were taken.

Since we were trying to find possible dye tracks, we used a probabilistic clustering method to identify aggregates of cells with high probabilities of dye presence. Two clusters were identified (Fig. 18). For each cluster, we propagated the probabilistic dye presence cell estimates using a stochastic interpolation method, leading to two dye "blob" hypotheses (Fig. 19).

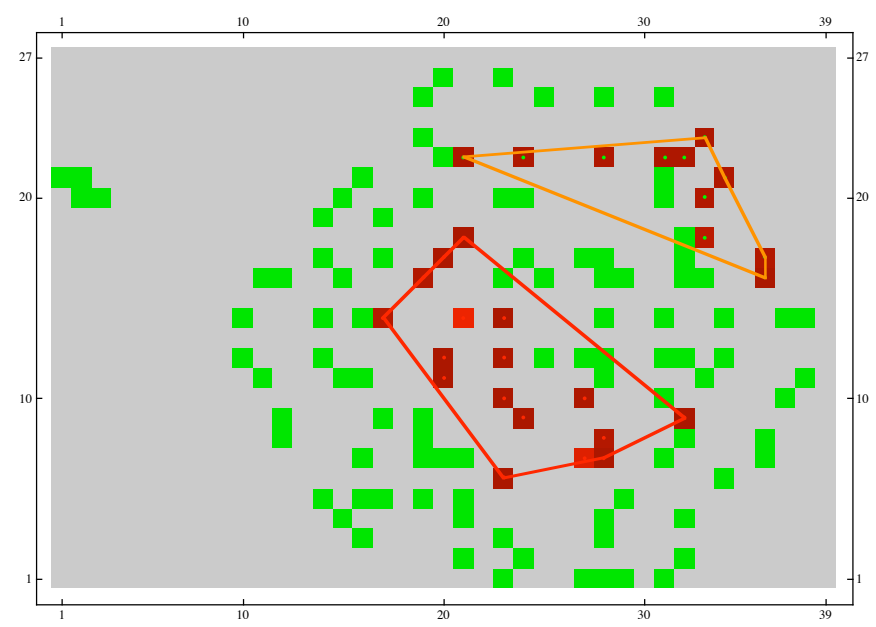

Fig. 18. Two clusters were identified in the PDM.

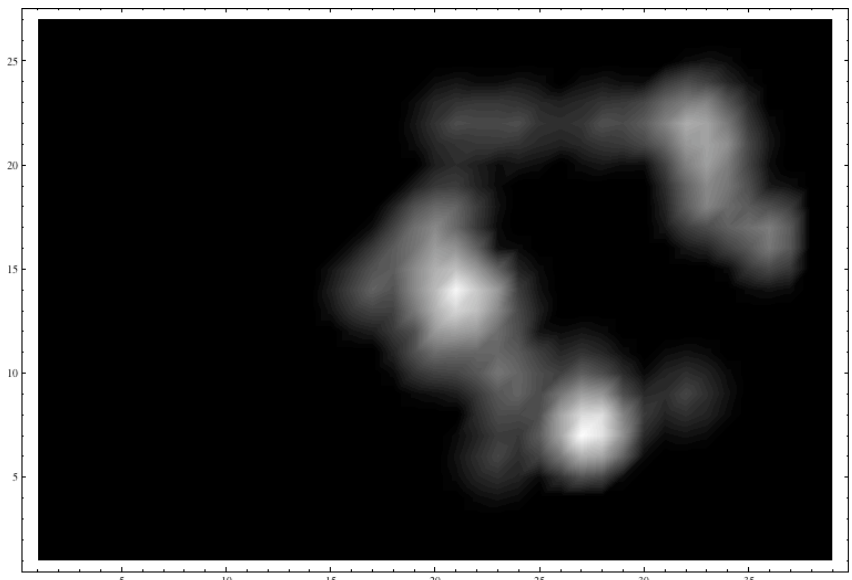

Fig. 19. Two dye track ("blob") hypotheses computed from the clusters identified in the PDM.

Manual comparison suggested the dye track hypotheses obtained may correspond to the tracks that can be visually identified in Fig. 20. We emphasize, however, that due to some data logging problems onboard the aerostat we have not been able to perform an automated comparison of the dye track hypotheses against visual ground truth. The latter is essential to allow us to assess the actual performance of the OASIS boats and the overall TAOSF system. We expect to be able to perform this automated comparison in future field tests.

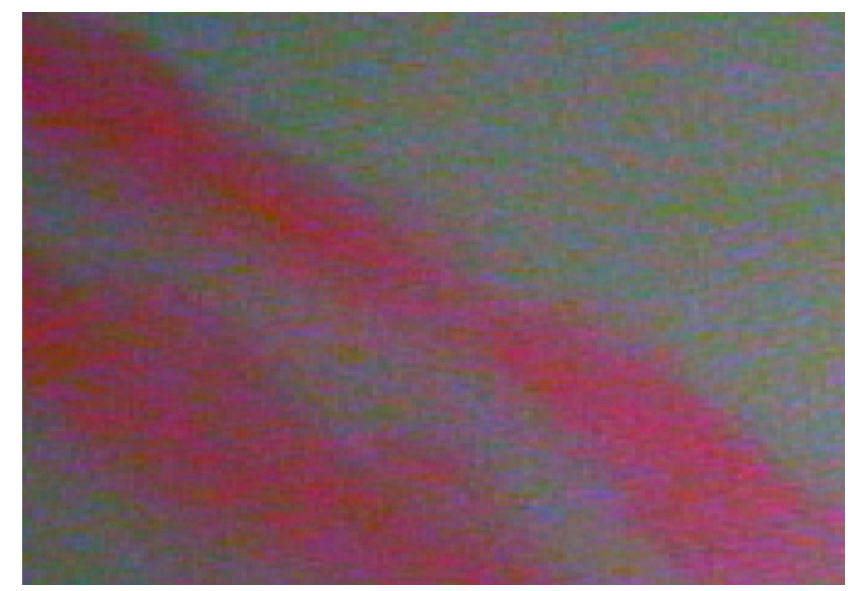

Fig. 20. Aerial image of the dye tracks in the region traversed by the OASIS vessel.

\section{CONCLUSIONS AND FUTURE WORK}

This paper describes a telesupervision architecture for multiple autonomous platforms and its application to the particular problem of the detection of harmful algal blooms. Initial work has concentrated on the integration of subsystems developed by the collaborating organizations, the development of HAB and dye observation systems, and the development of an aerial platform to allow observation of the TAOSF system and the OASIS boats from the air. Inwater tests have demonstrated the viability of components of the TAOSF architecture. 
One of our key next steps is an extended end-to-end test in which multiple OASIS platforms map a rhodamine-dye HAB surrogate under human telesupervision. Over the next year we plan to increase the number of platforms, develop and deploy adaptive sensing algorithms, and continue deploying the TAOSF system in the Chesapeake Bay estuary.

Because of their in situ observation capabilities and resolution, as well as their adaptivity, telesupervised autonomous surface vehicles are crucial to the sensor web for Earth science. The MARTA telesupervision architecture underlying TAOSF is broadly applicable to a wide variety of domains beyond $\mathrm{HABs}$, including ecological forecasting, water management, carbon management, disaster management, coastal management, homeland security, and planetary exploration.

\section{REFERENCES}

[1] G. Podnar, J. M. Dolan, A. Elfes, M. Bergerman, H. B. Brown, and A. D. Guisewite. "Human Telesupervision of a Fleet of Autonomous Robots for Safe and Efficient Space Exploration". Proceedings of the 1st Annual Conference on Human-Robot Interaction (HRI-2006), Salt Lake City, UT, USA, March 2-4, 2006.

[2] G. Podnar, J. Dolan, A. Elfes, and M. Bergerman. "Human Telesupervision of Very Heterogeneous Planetary Robot Teams". In Proceedings of the AIAA Space 2007 Conference, Long Beach, CA, 18 20 September 2007.

[3] A. Elfes, J. M. Dolan, G. Podnar, S. Mau, and M. Bergerman. "Safe and Efficient Robotic Space Exploration with Tele-Supervised Autonomous Robots." Proceedings of the AAAI Spring Symposium 2006, Stanford, CA, USA, March 27-29, 2006.

[4] Fish and Wildlife Research Institute. http://research.myfwc.com/

[5] Northwest Fisheries Science Center: Harmful Algal Blooms Program. http://www.nwfsc.noaa.gov/hab/index.html

[6] Mapping Harmful Algal Blooms in the Chesapeake Bay. http://coastwatch.noaa.gov/cbay_hab/

[7] J. M. Dolan, G. Podnar, S. Stancliff, E. Lin, J. Hosler, T. Ames, J. Moisan, T. Moisan, J. Higinbotham, and A. Elfes. "Harmful Algal Bloom Characterization via the Telesupervised Adaptive Ocean Sensor Fleet". Proceedings of the 2007 NASA Science and Technology Conference (NSTC-07), College Park, MD, June 19 - 212007.

[8] A. Elfes, J. Dolan and G. Podnar. "The Telesupervised Adaptive Ocean Sensor Fleet". In Proceedings of the Conference on Atmospheric and Environmental Remote Sensing Data Processing and Utilization III: Readiness for GEOSS, 2007 SPIE Symposium on Optical Engineering and Applications. San Diego, CA, 26 - 30 August 2007.

[9] Technical Data Sheet:

http://www.coleparmer.com/catalog/Msds/msdbes002.pdf

[10] A. Elfes. "Using Occupancy Grids for Mobile Robot Perception and Navigation". IEEE Computer Magazine, Special Issue on Autonomous Intelligent Machines, v. 22, n. 6, June 1989.

[11] A. Elfes. Multi-Source Spatial Fusion Using Bayesian Reasoning. In M. A. Abidi and R. C. Gonzalez (eds.), Data Fusion in Robotics and Machine Intelligence, Academic Press, San Diego, CA, 1992.

[12] A. Elfes. Dynamic Control of Robot Perception Using Stochastic Spatial Models. In G. Schmidt (ed.), Information Processing in Mobile Robots, Springer-Verlag, Berlin, July 1991.

[13] A. Elfes. "Robot Navigation: Integrating Perception, Environment Constraints and Task Execution Within a Probabilistic Framework". In
Reasoning with Uncertainty in Robotics, Springer-Verlag, Berlin, Germany, 1996.

[14] Bruch, M. H., "The multi-robot operator control unit (MOCU)," SPIE Proc. 6230: Unmanned Systems Technology VIII, Defense Security Symposium, Orlando, FL, April 18-20, 2006. 